\title{
Persistência de imazethapyr no solo e toxicidade ao milho semeado em sucessão
}

\author{
Persistence of imazethapyr in the soil and phytotoxicity to corn seeded as \\ succeeding crop
}

\section{Saul Jorge Pinto de Carvalho ${ }^{*}$, Daniel Jorge Soares ${ }^{2}$, Ramiro Fernando López Ovejero ${ }^{2}$, Pedro Jacob Christoffoleti ${ }^{3}$}

Resumo - Este trabalho foi desenvolvido com o objetivo de avaliar a persistência do herbicida imazethapyr no solo, bem como a toxicidade à cultura do milho cultivado em sucessão. Os tratamentos foram organizados em esquema fatorial com dois tipos de solo (S; argiloso e arenoso), três regimes de irrigação (I; diário, semanal e ausente) e quatro épocas (E) de aplicação do produto (90, 60 e 30 dias anteriores à semeadura do milho, além de testemunha sem aplicação). Para tanto, o herbicida imazethapyr foi aplicado na dose única de $100 \mathrm{~g} \mathrm{ha}^{-1}$. Foi adotado delineamento de blocos ao acaso com quatro repetições, totalizando 96 parcelas. Cada parcela foi caracterizada por um vaso de 8,5 L, preenchido com solo peneirado. Em síntese, a adequada disponibilidade hídrica entre a aplicação do produto e a semeadura do milho garantiu menores níveis de toxicidade à cultura e maior produção de massa de matéria seca. Em solo argiloso foram observados os menores níveis de toxicidade à cultura. A persistência do produto no solo foi reduzida sempre que ocorreu maior atividade microbiana, favorecida pela temperatura, disponibilidade de água no ambiente e, em menor grau, pelo tempo de persistência do produto no solo.

Palavras-chave: atividade residual, degradação microbiana, dinâmica, dissipação

Abstract - This work was carried out to evaluate imazethapyr persistence in the soil, as well as the phytotoxicity of this herbicide to the corn crop seeded in succession. Treatments were organized according to a factorial scheme with two types of soil (S; clay and sandy), three regimes of irrigation (I; daily, weekly and no irrigation) and four timing (T) of herbicide application (90, 60 and 30 days prior to corn seeding, besides check plots without application). For that, imazethapyr was applied at the unique rate of $100 \mathrm{~g} \mathrm{ha}^{-1}$. Experimental design of completely randomized blocks was adopted, with four replicates and 96 plots. Each plot consisted of a 8,5L plastic pot, fulfilled with sieved soil. In synthesis, adequate water availability between herbicide application and corn seeding granted the lowest levels of crop phytotoxicity and the highest yield of dry matter. In clay soil, the lowest levels of crop phytotoxicity were observed. Thus, it was possible to infer that imazethapyr persistence in the soil is reduced whenever there are higher microbial activity, which is enhanced by higher temperature, water availability and, in lower degree, by the time of herbicide persistence in the soil. Keywords: carryover, microbial degradation, dynamics, dissipation

Recebido: Outubro 16, 2017. Aceito: Junho 02, 2018.

${ }^{1}$ Instituto Federal do Sul de Minas Gerais, Professor Doutor Machado, Campus Machado, CP 1054, CEP 37750-971, Machado, MG, Brasil. E-mail: sjpcarvalho@yahoo.com.br

${ }^{2}$ Monsanto do Brasil, São Paulo, SP, Brasil. E-mail: daniel.j.soares@monsanto.com; ramiro.f.ovejero@monsanto.com

${ }^{3}$ Escola Superior de Agricultura "Luiz de Queiroz", Universidade de São Paulo, Piracicaba, SP, Brasil.

E-mail:pjchrist@usp.br 


\section{Introdução}

Reconhecidamente, a cultura da soja [Glycine max (L.) Merrill] tem posição de destaque na economia brasileira, com enorme importância para a balança comercial do país. Para plena produtividade da soja, há necessidade de se controlar os agentes bióticos presentes nas lavouras que possuem capacidade de interferir no crescimento e rendimento da cultura. Neste ambiente, destacam-se as plantas daninhas, devido à competição por água, luz e nutrientes, além de hospedarem pragas e doenças, o que resulta em significativas perdas de produção (Rizzardi et al., 2003; Agostinetto et al., 2014).

Neste contexto, o controle das plantas daninhas é prática obrigatória para assegurar a produtividade, com destaque para o controle químico por meio do uso de herbicidas. A grande adoção do método químico para controle de plantas daninhas foi consequência da maior tecnificação da agricultura brasileira, da descoberta de herbicidas cada vez mais eficientes e menos tóxicos, da capacidade de manejo em grandes áreas e em curto período de tempo e, principalmente, do baixo custo deste método (Silva et al., 1999; Agostinetto et al., 2014).

No Brasil, há mais de 30 moléculas de herbicidas com registro para uso na cultura da soja (Rodrigues e Almeida, 2011). Com o advento das culturas geneticamente modificadas para tolerância ao glyphosate e os sistemas conservacionistas de manejo do solo (plantio direto), o glyphosate rapidamente tornou-se o herbicida mais utilizado no mundo (Christoffoleti et al., 2008). Porém, o uso contínuo da mesma molécula, com várias aplicações anuais, por sucessivos anos, selecionou populações de plantas daninhas resistentes a este herbicida, como por exemplo as espécies de buva (Conyza spp.) (HEAP, 2015).

Quando uma população resistente de plantas daninhas é selecionada em determinada área, tem-se a necessidade de mudanças nas práticas utilizadas para seu manejo (Moreira et al., 2010). Segundo Peterson (1999), a mudança mais comum adotada pelos agricultores é a inclusão de herbicidas com mecanismos de ação alternativos no sistema de produção. No caso do manejo da buva na cultura da soja resistente ao glyphosate, com frequência, outras moléculas são utilizadas, com destaque para o imazethapyr.

O imazethapyr é um herbicida inibidor da ALS, com comportamento de ácido fraco com dois pontos de ionização, respectivamente com pKa de 2,1 e 3,9. Possui solubilidade de $1.400 \mathrm{mg} \mathrm{L}^{-1} \mathrm{e} \mathrm{K}_{\mathrm{ow}}$ influenciado pelo $\mathrm{pH}$ do meio $\left(\mathrm{K}_{\mathrm{ow}} 11, \mathrm{pH} 5 ; 31 \mathrm{pH} 7\right.$ e $16 \mathrm{pH}$ 9), o que indica alta solubilidade e pequena sorção aos coloides do solo. Sua via prioritária de degradação é microbiana, com $t_{1 / 2}$ no campo de 60 a 90 dias (Cantwell et al., 1989; Rodrigues e Almeida, 2011).

A semeadura do milho como segunda safra após a colheita da soja, também conhecido como milho safrinha, é uma realidade no Brasil (Artuzi e Contiero, 2006). Para tanto, tem-se observado a adoção de cultivares de soja de ciclo precoce e superprecoce, aliada a técnicas de antecipação da colheita. Por conta disso, o período de tempo entre a colheita da soja e a semeadura das culturas em sucessão é reduzido, elevando o risco de efeitos negativos causados pela atividade residual de herbicidas aplicados na soja sobre as culturas agrícolas semeadas em sucessão, na mesma área (Dan et al., 2012a).

Dentre os fatores que interferem na persistência de herbicidas no ambiente, pode-se destacar o teor de água no solo. A quantidade, a frequência e a intensidade das chuvas são importantes fatores que influenciam na maior ou menor atividade residual de herbicidas no solo, visto que a umidade interfere nos processos químicos e biológicos responsáveis pela dissipação dos herbicidas no ambiente (Silva et al., 1999). Segundo Ogg Júnior e Dowler (1988), a quantidade de água, proveniente de chuva ou irrigação, aplicada na área após pulverização do herbicida pode afetar a distribuição, movimento, persistência e eficácia do produto, assim como a tolerância da cultura ao herbicida. 
Assim sendo, considera-se a hipótese que o efeito residual do imazethapyr pode se manter no solo por período superior ao ciclo cultural, interferindo no milho cultivado em sucessão, sob influência do tempo de persistência no solo, regime de irrigações e tipo de solo. Este trabalho foi desenvolvido com o objetivo de avaliar a persistência de imazethapyr no solo, bem como a toxicidade à cultura do milho cultivado em sucessão, considerando-se dois tipos de solo, diferentes regimes de irrigação e intervalos entre a aplicação do herbicida e a semeadura do milho.

\section{Material e métodos}

$\mathrm{O}$ experimento foi desenvolvido em casa-de-vegetação, no município de Santa Cruz das Palmeiras - SP (21 ${ }^{\circ} 49^{\prime} 07^{\prime \prime}$ S, 47 $7^{\circ} 16^{\prime} 06^{\prime \prime} \mathrm{W}$ e $684 \mathrm{~m}$ de altitude), entre Março e Julho de 2010. Os tratamentos foram organizados segundo esquema fatorial triplo $2 \times 3 \times 4$; considerando-se dois tipos de solo (LATOSSOLO VERMELHO de textura argilosa e NEOSSOLO QUARTZARÊNICO de textura arenosa; Tabela 1); três regimes de irrigação: diário ( $5 \mathrm{~mm})$, intermitente com apenas uma irrigação semanal ( $5 \mathrm{~mm})$ e sem irrigação entre a aplicação do produto e a semeadura do

Tabela 1. Propriedades físico-químicas dos solos utilizados nos experimentos. Santa Cruz das Palmeiras - SP, 2010.

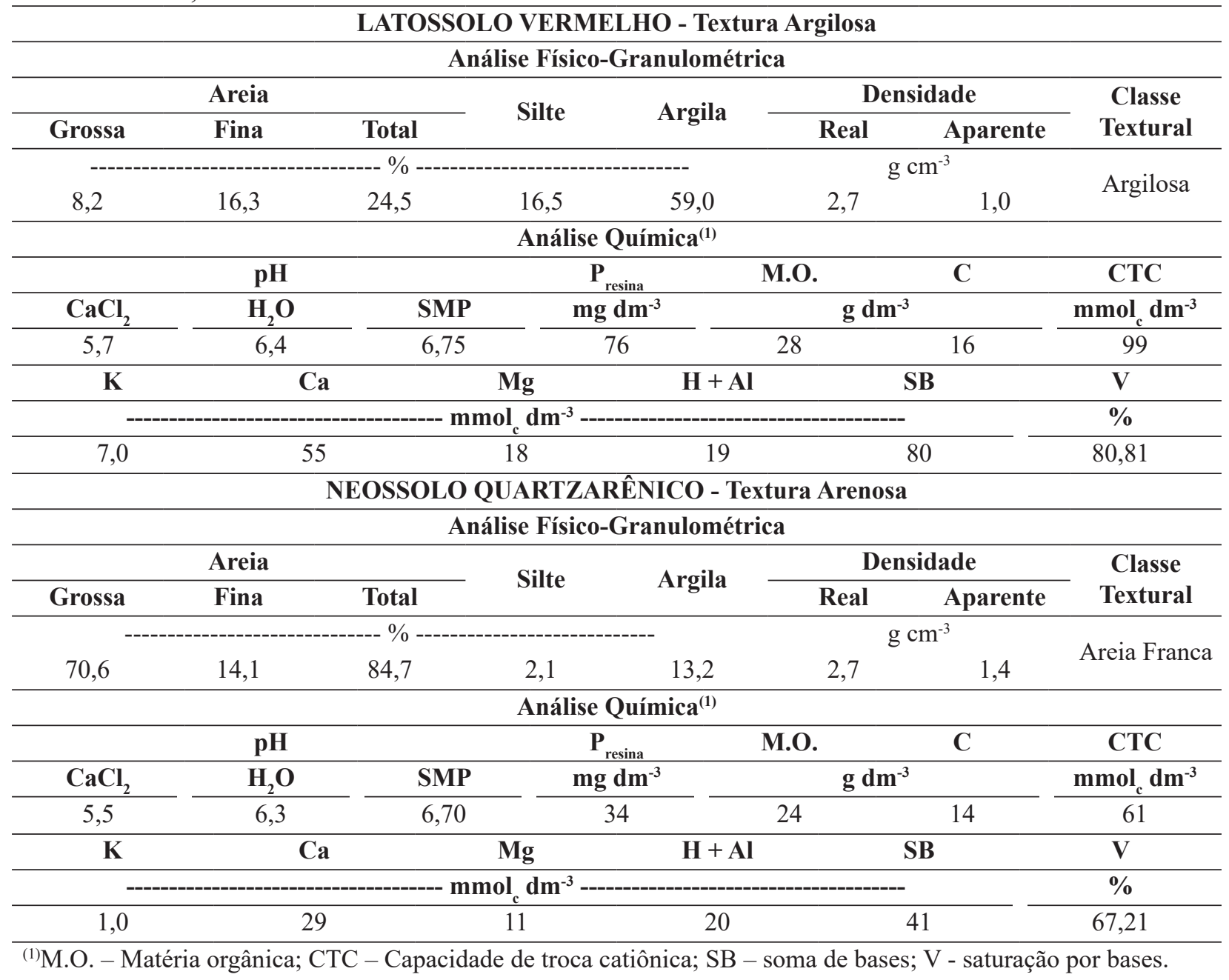


milho; e quatro épocas de aplicação do produto: 90,60 e 30 dias anteriores à semeadura (DAS) do milho e testemunha com ausência de aplicação.

Foi adotada uma aplicação de imazethapyr em dose de $100 \mathrm{~g} \mathrm{ha}^{-1}$, conforme recomendação de bula (Rodrigues e Almeida, 2011). A cultura do milho foi semeada em sucessão, utilizando-se o híbrido DKB390YGRR como bioindicador de toxicidade e persistência dos produtos no solo. As parcelas experimentais constaram de vasos plásticos com capacidade para $8,5 \mathrm{~L}$, preenchidos com solo argiloso ou arenoso, previamente peneirados e destorroados, de acordo com o esquema de tratamentos. Foi utilizado delineamento experimental de blocos ao acaso, com quatro repetições, resultando em 96 parcelas. As aplicações foram realizadas de forma escalonada, de modo que a semeadura do milho foi realizada na mesma ocasião, em densidade de seis sementes por vaso, na profundidade de $0,03 \mathrm{~m}$. Após a semeadura do milho todas as parcelas foram irrigadas diariamente até o final do trabalho.

Todas as aplicações foram realizadas com auxílio de um pulverizador costal pressurizado por $\mathrm{CO}_{2}$ acoplado à barra de pulverização com quatro pontas do tipo TT 110.015 , posicionada à $0,50 \mathrm{~m}$ do alvo, devidamente calibrada para volume de calda proporcional a $120 \mathrm{~L} \mathrm{ha}^{-1}$. Após semeadura do milho, avaliou-se a toxicidade das plantas aos 7 e 14 dias após a emergência (DAE), por meio da escala EWRC (1964), com intervalo de notas entre um e nove, em que um representou a ausência de sintomas e nove representou a morte das plantas. Aos 21 DAS, avaliou-se a massa de matéria seca do milho, coletando-se a parte aérea das plantas remanescentes nas parcelas. O material foi seco em estufa com circulação forçada a $60^{\circ} \mathrm{C}$, por 72 horas, até massa constante.

Os dados foram submetidos à aplicação do teste F na análise da variância, seguido de teste de Tukey, ambos com 5\% de significância. As testemunhas experimentais foram utilizadas para padronizar as avaliações de toxicidade, porém retiradas da análise estatística desta variável devido à ausência de notas. Quando se detectou interação fatorial, esta foi decomposta, analisando-se a participação dos fatores de tratamentos, também com 5\% de significância.

\section{Resultados e discussão}

Por meio do quadro da análise da variância, observam-se efeitos isolados dos fatores solo (S) e irrigação (I) para todas as variáveis; e somente a interação $\mathrm{S} x \mathrm{I}$ foi significativa para toxicidade aos 7 DAE e massa de matéria seca (Tabela 2). Neste caso, observou-se maior toxicidade absoluta em solo arenoso, nas avaliações de 7 e 14 DAE, bem como menor massa de matéria seca. Quanto à irrigação, tanto o regime diário de irrigação quanto o intermitente foram suficientes para garantir menor toxicidade e maior desenvolvimento das plantas (Tabela 2).

Segundo Loux et al. (1989), nas regiões produtoras de soja dos EUA, $80 \%$ da dissipação do imazethapyr ocorre até 60 dias após aplicação, porém foram necessários 160 dias para completa degradação da molécula. Assim, a persistência do imazethapyr é variável e influenciada pela umidade, temperatura e grau de sorção (Renner et al., 1988; Oliveira Júnior et al., 1999). A intensidade de sorção, por sua vez, é influenciada pela textura do solo, pelo teor de matéria orgânica e $\mathrm{pH}$, sendo mais intensa em pH mais ácido (Loux et al., 1989; Che et al., 1992; Oliveira Júnior et al., 1999).

Neste experimento, foram utilizados solos com $\mathrm{pH}\left(\mathrm{H}_{2} \mathrm{O}\right)$ superior a 6,3 (Tabela 1), o que reduziu a sorção do produto, aumentando sua concentração na solução do solo. Em campo, a lixiviação do imazethapyr é considerada baixa (Loux et al., 1988; Rodrigues e Almeida, 2011); assim sendo, o solo que tem maior capacidade de retenção de água também terá maior atividade de microrganismos e, por consequência, menor persistência do produto devido à degradação microbiana (Goetz et al., 1990; Carvalho et al., 2012). Por consequência, maior toxicidade foi observada em condição de semeadura em solo sem irrigação prévia (Tabela 3). 
Tabela 2. Quadro da análise da variância e comparação de médias ${ }^{1}$ para toxicidade (7 e 14 dias após a emergência (DAE)) e massa de matéria seca (21 DAE) de plantas de milho semeadas em solo previamente pulverizado com imazethapyr. Santa Cruz das Palmeiras - SP, 2010.

\begin{tabular}{cccc}
\hline Causa de Variação & Toxicidade 7 DAE & Toxicidade 14 DAE & Matéria Seca (g) \\
\hline Solo (S) & $* *$ & $*$ & $* *$ \\
Irrigação (I) & $* *$ & $* *$ & $* *$ \\
Época de Aplicação (E) & $\mathrm{ns}$ & $\mathrm{ns}$ & $\mathrm{ns}$ \\
S x I & $* *$ & $\mathrm{~ns}$ & $* *$ \\
S x E & $\mathrm{ns}$ & $\mathrm{ns}$ & $\mathrm{ns}$ \\
I x E & $\mathrm{ns}$ & $\mathrm{ns}$ & $\mathrm{ns}$ \\
S x I x E & $\mathrm{ns}$ & $\mathrm{ns}$ \\
\hline \multicolumn{5}{c}{ Tipo de Solo (S) } \\
\hline Argiloso & $2,3 \mathrm{~b}$ & $1,8 \mathrm{~b}$ & $3,77 \mathrm{a}$ \\
Arenoso & $2,9 \mathrm{a}$ & $2,2 \mathrm{a}$ & $2,56 \mathrm{~b}$ \\
\hline \multicolumn{5}{c}{ Irrigação (I) } \\
\hline Diária & $1,9 \mathrm{~b}$ & $1,6 \mathrm{~b}$ & $3,49 \mathrm{a}$ \\
Intermitente & $1,7 \mathrm{~b}$ & $1,1 \mathrm{~b}$ & $3,37 \mathrm{a}$ \\
Sem irrigação & $4,2 \mathrm{a}$ & $2,63 \mathrm{~b}$ \\
\hline \multicolumn{2}{c}{ Época de Aplicação (E) } \\
\hline Testemunha & ----- & 3,26 \\
30 DAS & 2,8 & 1,9 & 3,16 \\
60 DAS & 2,8 & 2,2 & 3,11 \\
90 DAS & 2,3 & 2,0 & 3,13 \\
\hline
\end{tabular}

${ }^{1}$ Médias seguidas por letras iguais, nas colunas, não diferem entre si segundo teste de Tukey, com 5\% de significância; ${ }^{2} \mathrm{DAS}$ - dias anteriores à semeadura; *Teste $\mathrm{F}$ significativo a $5 \%$ de probabilidade; **Teste $\mathrm{F}$ significativo a $1 \%$ de probabilidade; ns Não significativo.

Tabela 3. Toxicidade (7 $\left.\mathrm{DAE}^{1}\right)$ e massa de matéria seca $(21 \mathrm{DAE})$ de plantas de milho ${ }^{2}$ semeadas em solo previamente pulverizado com imazethapyr, influenciada pelo tipo de solo e pelo regime de irrigações. Santa Cruz das Palmeiras - SP, 2010

\begin{tabular}{|c|c|c|c|c|}
\hline \multirow{2}{*}{ Tipo de Solo (S) } & \multicolumn{4}{|c|}{ Regime de Irrigação (I) } \\
\hline & Diária $^{3}$ & & & Sem Irrigação \\
\hline \multicolumn{5}{|c|}{ Toxicidade aos 7 DAE } \\
\hline Arenoso & 2,7 & 1,8 & $\mathrm{aC}$ & 4,3 \\
\hline \multirow[t]{2}{*}{ Argiloso } & 1,1 & 1,6 & $\mathrm{aB}$ & 4,1 \\
\hline & $\mathrm{DMS}_{\text {Coluna }}=0,690$ & \multicolumn{2}{|c|}{$\mathrm{DMS}_{\text {Linha }}=0,830$} & $\mathrm{CV}(\%)=33,70$ \\
\hline \multicolumn{5}{|c|}{ Massa de Matéria Seca aos 21 DAE } \\
\hline Arenoso & 2,56 & 2,81 & $\mathrm{bA}$ & 2,28 \\
\hline \multirow[t]{2}{*}{ Argiloso } & 4,42 & 3,93 & $\mathrm{aB}$ & 2,97 \\
\hline & $\mathrm{DMS}_{\text {Coluna }}=0,293$ & $\mathrm{DM}$ & 352 & $\mathrm{CV}(\%)=13,12$ \\
\hline
\end{tabular}

${ }^{1}$ DAE - dias após emergência; ${ }^{2}$ Médias seguidas por letras iguais, maiúsculas nas linhas e minúsculas nas colunas, não diferem entre si segundo teste de Tukey, com $5 \%$ de significância; ${ }^{3}$ Diária - irrigação diária com 5 mm, Intermitente - irrigação semanal com $5 \mathrm{~mm}$; DMS - diferença mínima significativa; CV - coeficiente de variação.

Para solo argiloso, qualquer regime de irrigação foi suficiente para atenuar a intoxicação do milho. Em regime de irrigação diária, a toxicidade observada em solo arenoso foi superior àquela observada em solo argiloso, o que está relacionado com a via prioritária de degradação da molécula, por atividade microbiana. Solos mais arenosos possuem melhor drenagem e, por consequência, 
secagem mais rápida, o que gera ambiente menos favorável à comunidade microbiana quando comparado ao solo argiloso. Os dados de massa de matéria seca estão em concordância com a avaliação de toxicidade. A maior produção de massa de matéria seca foi obtida em solo argiloso irrigado diariamente (Tabela 3).

Também trabalhando com o imazethapyr, Dan et al. (2012b) relataram que a atividade residual desta molécula possui elevado potencial fitotóxico em plantas de girassol (Helianthus annuus) semeado em sucessão. Com frequência, tem-se observado o aparecimento de clorose internerval seguida de estrias foliares nas plantas. Sintomas similares foram descritos por Ulbrich et al. (1998) e Artuzi e Contiero (2006), após avaliarem a ação de imidazolinonas na cultura do milho. Por outro lado, Silva et al. (1999), em condição de campo, não observaram efeitos negativos de imazethapyr sobre plantas de sorgo, quando semeadas 120 ou 135 dias após a aplicação.

Estas observações sugerem que o fator de maior importância para dissipação de imazethapyr em solos agrícolas é a precipitação, que está diretamente relacionada com a atividade microbiana. Solos argilosos possuem maior potencial de retenção de água e, portanto, maior tempo para atividade de micro-organismos na degradação das moléculas, reduzindo a toxicidade quando sob irrigação. O solo arenoso, por sua vez, tem rápida drenagem, tornando-se seco mais rapidamente, o que resulta em menor atividade microbiana, ou seja, maior toxicidade.

\section{Conclusões}

O comportamento do imazethapyr no solo está diretamente relacionado à atividade microbiana, assim sendo, sua persistência será reduzida sempre que houver maior atividade microbiana, favorecida pela temperatura, disponibilidade de água no ambiente e, em menor grau, pelo tempo de persistência no solo.

\section{Referências}

Agostinetto, D.; Fontana, L.C.; Vargas, L.; Perboni, L.T.; Polidoro, E.; Silva, B.M. Competition periods of crabgrass with rice and soybean crops. Planta Daninha, v.32, n.1, p.31-38, 2014.

Artuzi, J.P.; Contiero, R.L. Herbicidas aplicados na soja e produtividade do milho em sucessão. Pesquisa Agropecuária Brasileira, v.41, n.7, p.1119-1123, 2006.

Cantwell, J.R.; Liebl, R.A.; Slife, F.W. Biodegradation characteristics of imazaquin and imazethapyr. Weed Science, v.37, n.6, p.815-819, 1989.

Carvalho, S.J.P.; Dias, A.C.R.; Minamiguchi, M.H.; Nicolai, M.; Christoffoleti, P.J. Atividade residual de seis herbicidas aplicados ao solo em época seca. Revista Ceres, v.59, n.2, p.278-285, 2012.

Che, M.; Loux, M.M.; Traina, S.J.; Logan, T.J. Effect of $\mathrm{pH}$ on sorption and desorption of imazaquin and imazethapyr on clays and humic acid. Journal of Environmental Quality, v.21, n.4, p.698-703, 1992.

Christoffoleti, P.J.; Galli, A.J.B.; Carvalho, S.J.P.; Moreira, M.S.; Nicolai, M.; Foloni, L.L.; et al. Glyphosate sustainability in South American cropping systems. Pest Management Science, v.64, n.4, p.422-427, 2008.

Dan, H.A.; Dan, L.G.; Barroso, A.L.L.; Oliveira Neto, A.M.; Guerra, N. Resíduos de herbicidas utilizados na cultura da soja sobre o milho cultivado em sucessão. Revista Caatinga, v.25, n.1, p.86-91, 2012a.

Dan, H.A.; Dan, L.G.M.; Barroso, A.L.L.; Procópio, S.O.; Oliveira Júnior, R.S.; Braz, G.B.P.; Alonso, D.G. Atividade residual de herbicidas usados na soja sobre o girassol cultivado em sucessão. Ciência Rural, v.42, n.11, p.1929-1935, 2012 b.

Goetz, A.J.; Lavy, T.L.; Gbur, E.E. Degradation and field persistence of imazethapyr. Weed Science, v.38, n.4/5, p.421-428, 1990. 
EWRC - European Weed Research Council. Report of 3rd and 4th meetings of EWRC. Committee of methods in weed research. Weed Research, v.4, n.1, p.88, 1964.

HEAP. The international survey of herbicide resistant weeds. Disponível em: <http:// www.weedscience.org/Summary/Species. aspx? WeedID=239>. Acesso em: 01 de jan. 2015.

Loux, M.M.; Liebl, R.A.; Slife, F.W. Availability and persistence of imazaquin, imazethapyr, and clomazone in soil. Weed Science, v.37, n.2, p.259-267, 1988.

Loux, M.M.; Liebl, R.A.; Slife, F.W. Adsorption of imazaquin and imazethapyr in soils, sediments and selected adsorbents. Weed Science, v.37, n.5, p.712-718, 1989.

Moreira, M.S.; Melo, M.S.C.; Carvalho, S.J.P.; Nicolai, M.; Christoffoleti, P.J. Herbicidas alternativos para controle de biótipos de Conyza bonariensis e C. canadensis resistentes ao glyphosate. Planta Daninha, v.28, n.1, p.167-175, 2010.

Ogg Júnior, A.G.; Dowler, C.C. Applying herbicides through irrigation systems. In: McWorther, C.G.; Gebhardt, M.R. (Eds.). Methods of applying herbicides. Champaign: WSSA, 1988. p.145-164.

Oliveira Júnior, R.S.; Koskinen, W.C.; Ferreira, F.A.; Khakural, B.R.; Mulla, D.J.; Robert, P.J.
Spatial variability of imazethapyr sorption in soil. Weed Science, v.47, n.2, p.243-248, 1999.

Peterson, D.E. The impact of herbicide-resistant weeds on Kansas agriculture. Weed Technology, v.13, n.3, p.632-635, 1999.

Renner, K.A.; Meggit, W.F.; Penner, D. Effect of soil $\mathrm{pH}$ on imazaquin and imazethapyr adsorption to soil and phytotoxicity to corn (Zea mays). Weed Science, v.36, n.1, p.78-83, 1988.

Rizzardi, M.A.; Fleck, N.G.; Riboldi, J.; Agostinetto, D. Ajuste de modelo para quantificar o efeito de plantas daninhas e época de semeadura no rendimento de soja. Pesquisa Agropecuária Brasileira, v.38, n.1, p.35-43, 2003.

Rodrigues, B.N.; Almeida, F.S. Guia de herbicidas. 5.ed. Londrina: Iapar, 2011. 697p.

Silva, A.A.; Oliveira Júnior, R.S.; Costa, E.R.; Ferreira, L.R.; Constantin, J.; Apoloni, D.K.M.; Oliveira, M.F. Persistência de herbicidas do grupo das imidazolinonas e efeitos sobre as culturas sucessoras de milho e sorgo. Acta Scientiarum, v.21, n.3, p.459-465, 1999.

Ulbrich, A.V.; Rodrigues, B.N.; Lima, J. Efeito residual dos herbicidas imazaquim e imazethapyr, aplicados na soja, sobre o milho safrinha. Planta Daninha, v.16, n.1, p.137-147, 1998. 